\title{
Trend in the co-occurrence of extreme daily rainfall in West Africa since 1950
} Juliette Blanchet ${ }^{1}$, Claire Aly ${ }^{1}$, Théo Vischel ${ }^{1}$, Gérémy Panthou ${ }^{1}$, Youssouph Sané ${ }^{2}$, and Mariane Diop Kane ${ }^{2}$

${ }^{1}$ Univ. Grenoble Alpes, CNRS, IRD, IGE, F-38000 Grenoble, France

${ }^{2}$ Physical Sciences Division, ANACIM, Dakar-Yoff, Senegal

\section{Key Points:}

- We study the co-occurrence of extreme daily rainfall in Senegal and Central Sahel.

- A change is found in the $80 \mathrm{~s}$ in both regions but with contrasting results.

- In Senegal, anisotropic co-occurrence is found before the 80s and isotropic cooccurence afterwards.

- In Central Sahel, anisotropy is found over the whole period, with greater extension after the 80 s.

- This provides qualitative indicators on change in size and propagation of the strongest storms. 


\section{Abstract}

We propose in this paper a statistical framework to study the evolution of the co-occurrence of extreme daily rainfall in West Africa since 1950. We consider two regions subject to contrasted rainfall regimes: Senegal and the Central Sahel. We study the likelihood of the $3 \%$ largest daily rainfall (considering all days) in each region to occur simultaneously and, in a 20-year moving window approach, how this likelihood has evolved with time. Our method uses an anisotropic max-stable process allowing us to properly represent the co-occurrence of daily extremes and including the possibility of a preferred direction of co-occurrence. In Senegal, a change is found in the 80s, with preferred co-occurrence along the E-50-N direction (i.e. along azimuth $50^{\circ}$ ) before the 80 s, and weaker isotropic co-occurrence afterwards. In Central Sahel, a change is also found in the 80s but surprisingly with contrasting results. Anisotropy along the E-W direction is found over the whole period, with greater extension after the 80s. The paper discusses how the co-occurrence of extremes can provide a qualitative indicator on change in size and propagation of the strongest storms. This calls for further research to identify the atmospheric processes responsible for such contrasted changes in storm properties.

\section{Introduction}

The Sahelian region is one of the world's region with the strongest signal of climate change over the last sixty years [Hulme et al., 2001; Dai et al., 2004; Gallego et al., 2015]. This African band makes the transition between the dry Saharan Desert towards the North and the wet Sudanian region towards the South. It experiences a monsoon period from June to September with very variable and intermittent rainfalls in the form of storms, called meso-scale convective systems [Mathon et al., 2002]. The occurrence and intensity of these storms condition the hydrology in the region [Lebel et al., 2003; Vischel and Lebel, 2007]. They can lead to situations of either droughts or floods, with large consequences for such a vulnerable population to climate hazard [Tarhule, 2005; Di Baldassarre et al., 2010].

Several studies have shown that the recent climate in the Sahelian region can be divided into three periods [e.g. Lebel and Ali, 2009; Nicholson, 2013]. The first one, from 1950 to 1969 , corresponds to a relatively humid period, which was followed by a long and intense drought period between the 1970s and 1980s. Since the 1990s the mean annual rainfall levels have slightly increased with respect to the dry period. This has often led to 
consider the last two decades as a recovery period [e.g. Nicholson, 2005; Sanogo et al., 2015]. However annual rainfall remains on average much lower it was during the humid period and it features a strong interannual variability. Moreover the recovery is not consistant over the region with some significant contrasts between the Central Sahel (Niger, Burkina, Eastern Mali), which has progressively become wetter, and the Western Sahel (Western Mali, Senegal), which has remained much in deficit [Lebel and Ali, 2009]. In the Central Sahel, the strong decadal variability has been associated with significant changes in the amount of rainfall at mesoscale. The rainfall deficit of the dry period is mainly attributable to a significant decrease in the frequency of storms with no significant decrease in intensity [Le Barbé et al., 2002; Bell and Lamb, 2006]. On the contrary, over the last two decades, the occurrence of storms did not increase significantly compared to the dry period, but the contribution of the most intense storms to the rainfall regime reached unprecedented levels [Panthou et al., 2014]. The fact that Sahelian rainfall has turned into a more extreme regime is in phase with the intensification of the water cycle expected at the global scale. The rise in intense storms in the Sahel was recently related to the global warming and is thus likely to be a consistent feature of the Sahelian rainfall regime over the long term [Taylor et al., 2017].

The present study brings new insight into the evolution of extreme precipitation in the Sahel through the statistical analysis of the co-occurrence of extreme daily rainfall, i.e. of daily rainfall exceeding some large level. The co-occurrence is studied for the period 1950-2014 over two contrasted regions in the Western (Senegal) and the Central Sahel. The results lead us to speculate on possible changes in intense storm properties in light of the decadal variability and regional disparities.

A suitable theoretical framework for assessing the frequency of rainfall exceeding some large level and their evolution is provided by univariate extreme value statistics [Coles, 2001]. Panthou et al. [2013] uses this framework to show temporal nonstationarity in the local occurrence of extremes in the Sahelian region, i.e. in their marginal distributions. However this gives only indication about the evolution of extreme rainfall at a given site. The spatial (i.e. surfacic) scale is, by definition, missing. To cope with the spatial variability of heavy rainfall, areal data are required, which is often lacking. Thus one usually resorts to the spatial interpolation of point data supplied by raingages [De Michele et al., 2002; Ceresetti et al., 2012; Panthou et al., 2014], whose quality for representing the most extreme events may be questioned [Delrieu et al., 2014]. 
Another way of looking upon the spatial variability of heavy rainfall is to study the probability of different locations to co-experience some extreme event. A suitable framework for this is provided by the theory of max-stable processes for spatial extremes [de Haan and Ferreira, 2006]. Max-stable processes have been used recently for studying the co-occurrence in threshold exceedances of wave height [Raillard et al., 2014], rainfall [Thibaud et al., 2013; Huser and Davison, 2014] or river discharges [Asadi et al., 2015]. However these studies assume temporal stationarity in the probability of co-occurrent extremes. Nonstationarity in the co-occurence of extreme temperature has been studied in Reich et al. [2014] but considering temporal co-occurrence, not spatial one. In a spatial context, it has been studied in Westra and Sisson [2011]; Shang et al. [2011]; Zhang et al. [2014] for extreme precipitation by using nonstationary marginal distributions in maxstable processes or in Nicolet et al. [2016]; Shaby and Reich [2012] by studying the evolution of the max-stable spatial dependence of extreme snowfall and extreme temperature, respectively. However these studies consider concomitance in annual maxima, whose explanatory power is rather limited because it only informs on whether large maxima tend to co-occur the same year, without accounting for their timing within the year. Studying trend in the co-occurrence of threshold exceedances aims precisely at overcoming this drawback.

The data are presented in Section 2 while Section 3 describes how is modeled and inferred the probability of concomitant exceedances, which is based on an anisotropic max-stable model. Section 4 assesses the probability of concomitant large exceedances in the two regions and their evolution since the 50s in a 20 -year moving window approach. Section 5 discusses the results through a possible link between changes in the probability of concomitant exceedances and changes in the properties of extreme storms.

\section{Data}

Two climatologically contrasted Sahelian regions are used in this study: Senegal and the Central Sahel. The instrumented area in Senegal covers a surface of about $600 \times$ $600 \mathrm{~km}^{2}$, as displayed in Figure 2. The network comprises 38 stations with daily measurements since 1950. The instrumented area in the Central Sahel is divided into two networks. The first one covers about $1200 \times 600 \mathrm{~km}^{2}$ and straddles Niger, Burkina Faso, Togo and Benin (Figure 2). 44 stations are dispatched over this area, with daily measurements since 1950. The second network is the AMMA-CATCH rainfall network in Niger [Lebel 
et al., 2009]. It covers about $110 \times 120 \mathrm{~km}^{2}$ in the region of Niamey. It comprises 30 stations with 5' measurements since 1990, which we aggregate at daily scale.

We restrict the analysis to the four monsoon months of June-July-August-September (JJAS) and to the period 1950-2014, which is the period presenting the most complete data. The mean percentage of missing data over this period is $4 \%$ in Senegal, with a maximum of $15 \%$, and $3 \%$ in the Central Sahel, with a maximum of $12 \%$. Considering all pairs of stations, the mean percentage of days with at least one concurrent missing value is $7 \%$ in Senegal and 6\% in the Central Sahel. However more and more lack is found since the 90s. The Senegalese network comprises 38 stations with less than $15 \%$ of missing data in the 20-year window 1950-1969, but this decreases to 23 stations in 1995-2014. Similarly, the Central Sahelian network comprises 44 stations with less than $15 \%$ of missing data in 1950-1969 but 34 such stations in 1995-2014. In the moving window study of Section 4.2, we will only consider, for a given window, the stations comprising less than $15 \%$ of missing data over this window, meaning that actually less stations will be used in the most recent period to document co-occurrence. A separate analysis restricted to the stations having less than $15 \%$ of missing data on every 20 -year window (23 stations in Senegal and 30 stations in the Central Sahel) shows that the results of the present study are not dependent on the evolution of the network though years (not shown). For the small-scall network of AMMA-CATCH, the 1990-2014 period is considered. Within this period, each station has less than $10 \%$ of missing data. Due to the shorter measurement period, these data will not be considered in the non-stationarity study of Section 4.2 , but the higher spatial density of the network will provide valuable information of cooccurrence for small interdistances in Section 4.1.

Daily rainfall in JJAS can be considered as identically distributed. Moreover the little extension (less than 10km large) and short duration (maximum a few hours) of the strong convective cells induce a very strong spatio-temporal variability of rainfall [see Figure 4 of Vischel and Lebel, 2007, for example]. This implies in particular that daily amounts can be considered as almost independent from one day to another, as validated in Ali et al. [2006] and Gerbaux et al. [2009] for reproducing the interannual and intraseasonal variability of Sahelian rainfields. Serial independence of extremes -which are the main object of this study- can be quantitatively assessed by computing the mean number of consecutive values that exceed high thresholds. Independent exceedances correspond to a mean value of 1 and the closer to 1 , the less dependent the exceedances. Using the es- 
timation method of Ferro and Segers [2003], we find that the average mean cluster size is 1.2 in Senegal, 1.14 in Central Sahel and 1.05 for AMMA-CATCH network, which confirms that assuming independence of threshold exceedances is reasonable for the three networks.

As shown in Figure 2, a drawback of the studied networks might be that that none of them samples uniformly the space and the azimuths. The Sahelian stations are not uniformly distributed over the region, with a bigger density of stations close to the Atlantic coast. Stations of the Central Sahelian network are fairly uniformly dispatched but the region features a rectangular shape, with a N-S extension more limited than the E-W one. However a separate analysis performed on a subset of stations distributed quite uniformly on squared domains in both regions showed that actually the results of this study are quite robust to the shape and spatial distribution of the networks (not shown).

\section{Method}

\subsection{Probability of interest}

Let $X_{j t}$ be the random variable of daily rainfall at station $j$ and day $t$, for $t=1, \ldots, T$. Daily rainfalls $X_{j 1}, \ldots, X_{j T}$ are assumed to be independent and identically distributed, i.e. to be a random sample of a variable $X_{j}$ representing daily rainfall a given day at station $j$, with distribution function $F_{j}$.

Let $\mathcal{R}$ be a given region. We are interested in the probability of concomitant exceedance across $\mathcal{R}$, i.e. in

$$
\operatorname{pr}\left(X_{j}>x_{j} \text { for all } j \in \mathcal{R}\right)
$$

for $x_{j}$ s large. Eq. (1) is the probability that a large level is exceeded simultaneously everywhere across $\mathcal{R}$, due to a very convective activity making storms to affect all of $\mathcal{R}$ the same day. By large we mean here larger than the $\alpha$-quantile of the marginals distributions, i.e. for $x_{j}>u_{j}=F_{j}^{-1}(\alpha)$ with $\alpha$ large (e.g. $\alpha=0.97$ in Section 4). Note that knowing (1) for all $x_{j}$ large is equivalent to knowing

$$
\operatorname{pr}\left(h_{j}\left(X_{j}\right)>x_{j} \text { for all } j \in \mathcal{R}\right)
$$

for all $x_{j}$ large, with $h_{j}$ any positive bijective function. It is convenient to consider $h_{j}$ so that all $h_{j}\left(X_{j}\right)$ have the same margins. In particular, with $h_{j}()=.-1 / \log \left\{F_{j}().\right\}$, the trans- 
formed variables

$$
Y_{j}=h_{j}\left(X_{j}\right)
$$

are unit-Fréchet distributed, i.e. $\operatorname{pr}\left(Y_{j} \leq y\right)=e^{-1 / y}$. This framework turns out to be convenient to work with for spatial extremes, so in the rest of this paper we will be interested in the probability of simultaneous exceedance for the transformed variables $Y_{j}$, i.e.

$$
\operatorname{pr}\left(Y_{j}>y_{j} \text { for all } j \in \mathcal{R}\right)
$$

for all $y_{j}$ s large. The probability (1) is then simply obtained as (4) taken in $y_{j}=-1 / \log F_{j}\left(x_{j}\right)$.

By transforming the $X_{j}$ s into $Y_{j}$ s and studying (4) instead of (1), we assume that the marginal distributions $F_{j}$ s are known. Actually, since we are interested in (1) when all $x_{j}$ are larger than some large quantile $u_{j}$, only the tail of the marginal distribution is needed. The theory of univariate extremes (Coles [2001]) tells us that if $u_{j}$ is large enough, we can assume that peaks-over-threshold follow a GPD (Generalized Pareto Distribution), i.e. that for $x>u_{j}$

$$
F_{j}(x)=\operatorname{pr}\left(X_{j} \leq x\right)=1-(1-\alpha)\left(1+\xi_{j} \frac{x-u_{j}}{\sigma_{j}}\right)^{-1 / \xi_{j}}
$$

where $\sigma_{j}>0$ is the scale parameter and $\xi_{j}$ is the shape parameter. Thus a first step of our analysis is to estimate, for each station $j$, the GPD parameters $\sigma_{j}$ and $\xi_{j}$ based on the data exceeding the chosen threshold $u_{j}$, then use these estimates to transform the data $x_{j}$ exceeding the threshold into $y_{j}=-1 / \log F_{j}\left(x_{j}\right)$. The data below the threshold are set to $v=-1 / \log \alpha$ and tagged as "censored". They will be used to estimate the model (4) in a censored likelihood approach; see Section 3.2.4.

\subsection{Modeling of co-occurrence}

\subsubsection{Asymptotic dependence and asymptotic independence}

Here we describe how is modeled the probability of co-exceeding some large thresholds (1). This implies modeling the dependence between variables at large levels. Dependence models for extremes can be split into two families [Davison et al., 2013]: asymptotic dependence models and asymptotic independence models. Asymptotic independence models assume dependence of variables at moderate levels but independence as the levels approach the supremum of the support of the distribution. In particular the probability of one variable being larger than its $\eta$-quantile conditionally on another variable being larger 
than its $\eta$-quantile,

$$
\chi_{i j}(\eta)=\operatorname{pr}\left(X_{j}>F_{j}^{-1}(\eta) \mid X_{i}>F_{i}^{-1}(\eta)\right)
$$

converges to 0 as $\eta \rightarrow 1$, whatever the dependence at sub-extremal levels (provided the two variables are not almost surely equal), as illustrated Figure 1. The Gaussian dependence model, for example, belongs to this class as soon as its correlation coefficient is not 1, no matter how close to 1 [Coles et al., 1999]. Asymptotic dependence models, on the contrary, assume that some dependence remains at infinite levels, i.e. that $\chi_{i j}(\eta)$ converges to a positive value $\chi_{i j}^{+}$as $\eta \rightarrow 1$, as illustrated Figure 1. In practice distinguishing between weakly asymptotically dependent variables $\left(\chi_{i j}^{+}\right.$close to 0$)$ and asymptotically independent variables reveals difficult [Coles et al., 1999], and this is confirmed on our data. A separate analysis revealed that, when considering annual maxima, the asymptotically dependent Brown-Resnick process (see Section 3.2.2) and the asymptotically independent version of Wadsworth and Tawn [2012] are actually pretty equivalent. Comparison of the values of CLIC [Davison et al., 2013] in the stationary and non-stationary cases gave preference to either model depending on the period, in equal proportion, with always very similar values of CLIC. Indeed the estimated probabilities of co-occurrence, e.g. of $\chi_{i j}(\eta)$ in (6), are pretty similar whatever model when $\eta$ lies in the observed range of values, making selection difficult. Extrapolation at much larger levels obviously differ but according to CLIC no clear preference is given to either family of models. In this article, we have chosen to consider the asymptotically dependent case, discarding asymptotically independent models for the sake of concision, but also because, from a physical point of view, we don't see why more and more extreme storms should be less and less extended, as assumed by asymptotically independent models. However we keep in mind that our choice may not be optimal at extrapolation, and so that estimations of e.g. $\chi_{i j}(\eta)$ might be only valid in the observed range of values, in which case the choice of model seems to have little impact.

\subsubsection{Brown-Resnick model}

Let assume that the daily rainfall variables $X_{j} \mathrm{~s}$, or equivalently the $Y_{j} \mathrm{~s}$ of (3), are asymptotically dependent. Following de Haan and Ferreira [2006], chapter 9, well-founded asymptotically dependent models for maxima belong to the family of max-stable processes. Thibaud et al. [2013] justify that this theory can be used for large exceedances. Various parametric models of max-stable processes have been proposed in the literature. The most popular ones are the Smith process, the Schlather process [Schlather, 2002], the 

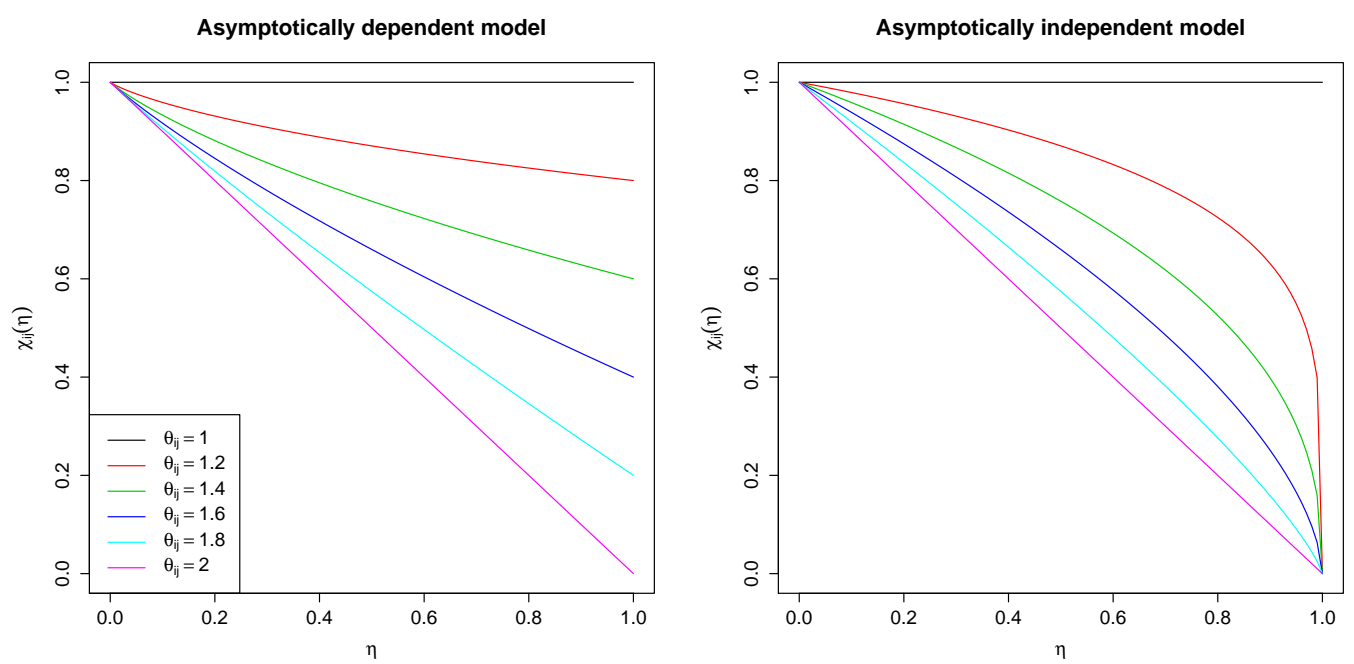

Figure 1. Theoretical conditional exceedance probabilities $\chi_{i j}(\eta)=\operatorname{pr}\left(X_{j}>F_{j}^{-1}(\eta) \mid X_{i}>F_{i}^{-1}(\eta)\right)$ for (left) an asymptotically dependent model corresponding to a max-stable process with extremal coefficient $\theta_{i j}=1$, $1.2,1.4,1.6,1.8,2$; (right) an asyptotically independent model constructed as the inverted max-stable process of the left panel [Wadsworth and Tawn, 2012].

Brown-Resnick process [Kabluchko et al., 2009], the Geometric Gaussian process [Davison et al., 2012] and the Extremal-t process [Opitz, 2013]. Few comparative studies exist in the literature but the overall consensus is to prefer the Brown-Resnick, Extremal-t and Geometric Gaussian processes towards the Smith and Schlather processes [Davison et al., 2012; Gaume et al., 2013], with actually very similar performances for the three first ones but a slightly better robustness of the Brown-Resnick process [Nicolet et al., 2017]. This lead us to consider the Brown-Resnick process for this study. Alternative options to maxstable modeling could be to consider generalized Pareto processes [Ferreira and de Haan, 2014; Dombry and Ribatet, 2015; Thibaud and Opitz, 2015].

Huser and Davison [2013] derive a closed-form expression for the multidimensional joint distribution of Brown-Resnick process. In particular, the bivariate distribution function is given by [Kabluchko et al., 2009]:

$$
\operatorname{pr}\left(Y_{i} \leq y_{i}, Y_{j} \leq y_{j}\right)=\exp \left\{-\frac{1}{y_{i}} \Phi\left(\frac{a_{i j}}{2}+\frac{1}{a_{i j}} \log \frac{y_{j}}{y_{i}}\right)-\frac{1}{y_{j}} \Phi\left(\frac{a_{i j}}{2}+\frac{1}{a_{i j}} \log \frac{y_{i}}{y_{j}}\right)\right\},
$$

for $y_{i}$ and $y_{j}>v$, where $\Phi$ is the standard Gaussian cumulative distribution function, $a_{i j}=\left\{2 \gamma\left(\left\|s_{i}-s_{j}\right\|\right)\right\}^{1 / 2}$, with $\gamma$ the (unknown) semivariogram of an intrinsically Gaussian process, and $\left\|s_{i}-s_{j}\right\|$ the distance between stations $i$ and $j$. Usual practice is to use the 
isotropic semivariogram,

$$
\gamma\left(\left\|s_{i}-s_{j}\right\|\right)=\left(\frac{\left\|s_{i}-s_{j}\right\|}{\lambda}\right)^{\kappa},
$$

with $\lambda>0$ and $0<\kappa \leq 2$ unknown parameters to estimate.

Combining (3) and (7) gives that, for any $\eta \geq \alpha$, the probability (6) of one variable being larger than its $\eta$-quantile conditionally on another variable being larger than its $\eta$ quantile is

$$
\chi_{i j}(\eta)=\left(1-2 \eta+\eta^{\theta_{i j}}\right)(1-\eta)^{-1},
$$

as illustrated Figure 1.

The scalar $\theta_{i j}$ in (9) is defined by $\theta_{i j}=2 \Phi\left\{\gamma\left(\left\|s_{i}-s_{j}\right\|\right)^{1 / 2} 2^{-1 / 2}\right\} \in[1,2]$ and is termed the extremal coefficient. For the semivariogram $\gamma$ defined by (8),

$$
\theta_{i j}=2 \Phi\left(\left\|s_{i}-s_{j}\right\|^{\kappa / 2} \lambda^{-\kappa / 2} 2^{-1 / 2}\right) .
$$

Whatever choice of $\gamma$, when $\theta_{i j}=1, X_{i}$ and $X_{j}$ are completely dependent, and $\chi_{i j}(\eta)$ in (9) is uniformly 1 . When $\theta_{i j}=2, X_{i}$ and $X_{j}$ are independent and $\chi_{i j}(\eta)=1-\eta$. Besides, whatever $\theta_{i j} \in[1,2]$,

$$
\chi_{i j}^{+}:=\lim _{\eta \rightarrow 1} \chi_{i j}(\eta)=2-\theta_{i j} .
$$

Actually, whatever $\theta_{i j}, \chi_{i j}(\eta)$ can be approximated by $\chi_{i j}^{+}$with less than $10^{-2}$ error as soon as $\eta \geq 0.99$, and in particular for the JJAS season as soon as we consider exeedances of the yearly return level. However, let us recall that extrapolation of $\chi_{i j}(\eta)$ beyond the range of observed values is very uncertain for our data because it relies on the assumption of asymptotic dependence, which we were unable to clearly validate. Thus, rather than a limit at infinite levels, $\chi_{i j}^{+}$will be interpreted in the rest of this article as the conditional probability of concomitant large exceedances, the term "large" embracing roughly the range of observed values (i.e. up to about the 100-year return level).

\subsubsection{Accounting for anisotropy}

According to (10) combined with (9), the Brown-Resnick is an isotropic model of concomitance: any two pairs of stations at the same distance apart have the same conditional probability of concomitant exceedance, whatever $\eta$. This means that the set of sites $s_{j}$ such that $\chi_{i j}(\eta)=p$, are circles centered on station $i$. However assumption of isotropy is often violated for hydrometeorological variables, which tend to show increased dependence in the direction of the governing wind fields [Ali et al., 2003; Blanchet and Davison, 
2011; Gaume et al., 2013; Nicolet et al., 2016]. In order to account for such anisotropy, we replace in (8) and (10) the isotropic distance by the anisotropic distance defined as

$$
\left\|s_{i}-s_{j}\right\|^{2}=\left(s_{j}-s_{i}\right)^{\prime} M^{\prime} M\left(s_{j}-s_{i}\right)
$$

where $s_{i}$ and $s_{j}$ are 2D-coordinates vectors, $s^{\prime}$ is the transpose of $s$ and

$$
M=\left(\begin{array}{cc}
\cos \psi & \sin \psi \\
-b \sin \psi & b \cos \psi
\end{array}\right)
$$

with $b>1$ the elongation coefficient and $\psi \in\left[-\frac{\pi}{2}, \frac{\pi}{2}\right]$ the angle. Now the set of sites $s_{j}$ such that $\chi_{i j}(\eta)=p$ are ellipses centered on station $i$, with the major axis oriented along $\psi$ and an elongation (ratio of the major and minor axes) equal to $b$.

\subsubsection{Inference}

Model (7) involves four parameters: $\lambda$ and $\kappa$ driving the strength of dependence along the major axis at a fixed distance, $b$ driving the strength of anisotropy and $\psi$ giving the direction of major dependence. Assuming temporal independence of the $y$ 's, but spatial dependence, the log-likelihood of the model is written:

$$
\ell(\lambda, \kappa, b, \psi)=\sum_{t=1}^{T} \log g\left(y_{1 t}, \ldots, y_{N t}\right)
$$

where $g$ is the multivariate density of Brown-Resnick model. Wadsworth and Tawn [2014] gives a closed form expression for $g$, however its computation results in a combinatorial explosion [Davison and Gholamrezaee, 2011; Castruccio et al., 2016]. It is possible to circumvent this issue by making estimation based on the pairwise log-likelihood [Varin et al., 2011; Padoan et al., 2010]

$$
\ell_{1}(\lambda, \kappa, b, \psi)=\sum_{t=1}^{T} \sum_{i=1}^{N-1} \sum_{j=i+1}^{N} \log g_{i j}\left(y_{i t}, y_{j t}\right),
$$

where $g_{i j}$ is the bivariate density of $\left(Y_{i}, Y_{j}\right)$, i.e. associated to (7), and $N$ is the number of stations.

In applying pairwise likelihood we must account for the fact that exceedances may occur in both variables, in one variable or in neither, whereas the bivariate density associated to (7) is only valid when both variables exceed the threshold $v$. To do so we apply the censoring approach described by Coles [2001], Section 8.3.1, and used in the context of threshold exceedances of spatial extremes in Thibaud et al. [2013]; Bacro and Gaetan 
[2014]; Huser and Davison [2014]; Raillard et al. [2014], for example. Writing $G_{i j}$ the bivariate distribution, valid only when both variables exceed $v$, the likelihood contribution of sites $i$ and $j$ in (15) is:

$$
g_{i j}\left(y_{i t}, y_{j t}\right)= \begin{cases}\partial_{i} \partial_{j} G_{i j}\left(y_{i t}, y_{j t}\right), & y_{i t}>v, y_{j t}>v ; \\ \partial_{i} G_{i j}\left(y_{i t}, v\right), & y_{i t}>v, y_{j t} \leq v ; \\ \partial_{j} G_{i j}\left(v, y_{j t}\right), & y_{i t} \leq v, y_{j t}>v ; \\ G_{i j}(v, v), & y_{i t} \leq v, y_{j t} \leq v ;\end{cases}
$$

$\partial_{i} G_{i j}$ denotes the differentiation with respect to $y_{i}$ in (7). No analytical expression for the maximum of (15) under (16) is available but maximization may be performed numerically (e.g. quasi-Newton method).

\subsection{Workflow}

Two frameworks are considered in this study. The first one assumes stationarity over the observation period. The workflow consists of the following steps:

- For each station $j$, the empirical $\alpha$-quantile is computed. The marginal GPD distribution $F_{j}$ above this threshold is estimated by maximum likelihood.

- The data $x_{j t}$ exceeding the threshold are transformed into unit-Fréchet variates $y_{j t}=-1 / \log F_{j}\left(x_{j t}\right)$. For the data $x_{j t}$ below the threshold, the exact value of $y_{j t}$ is not necessary since to compute (16) one only needs to know that $y_{j t}$ is in this case below $v$.

- The anisotropic Brown-Resnick model for the region $\mathcal{R}$ of interest is estimated based on the $\left\{y_{j t}, j \in \mathcal{R}, t=1, \ldots T\right\}$, by maximizing the pairwise log-likelihood (15) under (7) with censoring (16).

This gives one anisotropic Brown-Resnick model for each of the three networks. Determining the threshold above which this workflow may be applied is a bias-variance tradeoff. Too low a threshold is likely to violate the assumptions of extreme value theory, leading to bias. Too high a threshold implies too few excesses to estimate the model, leading to high variance. In this article, the threshold is set to the empirical $97 \%$ quantile of daily rainfall at each station, i.e. $\alpha=0.97$. This is slightly higher than the thresholds of Thibaud et al. [2013] (90\%-quantile) and Huser and Davison [2014] (95\%-quantile) for example but we use here longer time-series, so the number of exceedances is roughly similar. Setting the threshold to the 95\%-quantile leads anyway to similar results (not shown). 
The second framework considers 20-year moving windows from 1950-1969 to 19952014. The workflow consists of the same steps but applied to each window separately (i.e. considering only the days $t$ within the window). This means that the threshold is recomputed for each window and thus different thresholds are used for different windows. However the models considered here rely on asymptotic theory, so they should apply equally for any large enough threshold. We checked that using for each window the $97 \%$ quantile of the entire period does not affect the results, but this implied considering different amounts of data depending on the window, which is not recommended for model comparison and in particular for what matters uncertainty, so we prefer showing results of the window-dependent threshold case. Applying the above workflow gives us one BrownResnick model for each network and each window. Given the shortness of its observation period (25 years), the moving-window framework is not applied to the AMMA-CATCH network.

\section{Results}

\subsection{Stationary case}

\subsubsection{Marginal distributions}

Although marginal distributions are not the scope of this paper, they are interesting to study to better understand the climatology of rainfall in the region. Figure 2 shows the maps of (a): mean annual rainfall, (b): mean proportion of rainfall days, (c): mean of non-zero daily rainfall and (d): 50-year return levels in Senegal and Central Sahel in JJAS season, for the period 1950-2014. For the three former cases, empirical values are plotted while the 50 -year return level at station $j$ is given by $\hat{F}_{j}^{-1}(1-1 /(K L))$, where $L=122$ is the number of days in the JJAS season per year, $K=50$ and $\hat{F}_{j}$ is an estimate of the marginal distribution $F_{j}$ at station $j$. Values for the AMMA-CATCH network are not shown on this figure because the observation period is much shorter (1990-2014). Let just note that the average values for AMMA-CATCH network are of the same order as the values found in Niamey region in Figure 2. For example the mean 50-year return level for AMMA-CATCH network is around $110 \mathrm{~mm}$.

The 50-year return levels (d) in both Senegal and the Central Sahel show a N-S increase, although in Senegal the distance to the ocean seems also to play a role with larger values when moving closer to the Atlantic West coast. The 50-year return levels in Sene- 
(a)
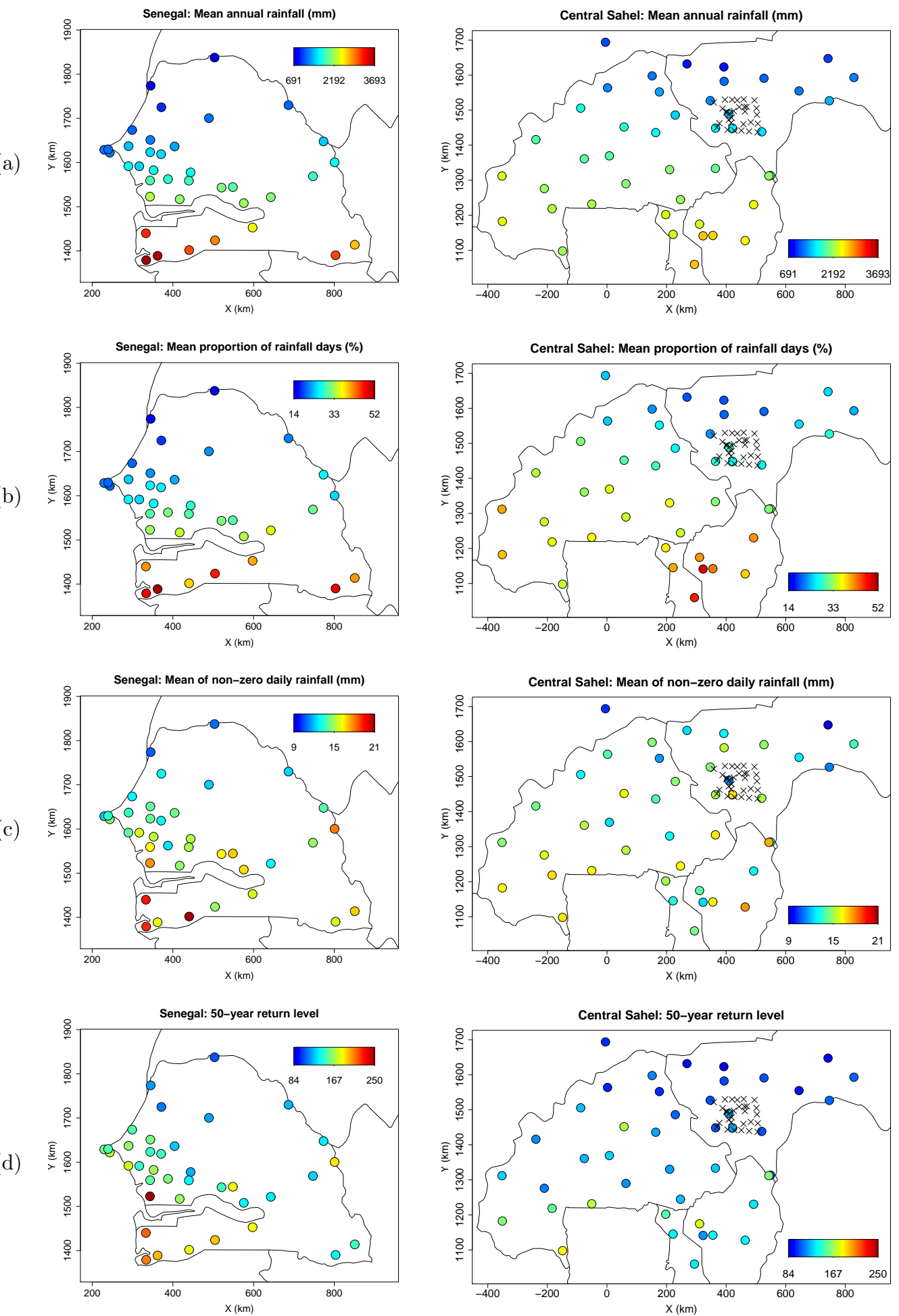

Figure 2. (a): Annual rainfall, (b): proportion of rainfall days, (c): mean of non-zero daily rainfall and (d): 50-year return level of daily rainfall accumulation $(\mathrm{mm})$ in Senegal and Central Sahel in JJAS season, for the period 1950-2014. The black crosses in the Central Sahelian map show AMMA-CATCH network. Coordinates are UTM coordinates. 
Senegal

Central Sahel
(a)
(b)
(c)
(d)
(a) (b)
(c)
(d)

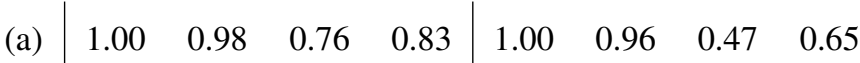

\begin{tabular}{lllll|llll} 
(b) & 0.98 & 1.00 & 0.64 & 0.71 & 0.96 & 1.00 & 0.23 & 0.43
\end{tabular}

\begin{tabular}{lllll|llll} 
(c) & 0.76 & 0.64 & 1.00 & 0.96 & 0.47 & 0.23 & 1.00 & 0.94
\end{tabular}

\begin{tabular}{lllll|llll} 
(d) & 0.83 & 0.71 & 0.96 & 1.00 & 0.65 & 0.43 & 0.94 & 1.00
\end{tabular}

gal are on average $20 \%$ larger than in the Central Sahel. This superiority applies actually for all $K \geq 1$ year, with greater differences as the return period increases. The 50 -year return level map in the Central Sahel shows a similar pattern to the 20- and 100- year return level maps produced in Panthou et al. [2012] over the same area but considering annual maxima. The fact that it differs from the 50-year return level map of Senegal both in terms of magnitude and shape is a novel result.

As shown in Table 1, the proportion of rainfall days (b) explains more the annual totals (a) than the mean of non-zero daily rainfalls (c), as already noted in Le Barbé and Lebel [1997]; Le Barbé et al. [2002]. The return level map of the Central Sahel (d) is more correlated to the proportion of rainfall days (b) than to the mean of non-zero daily rainfalls (c), which is more patchy. This suggests that the N-S increase of extreme rainfall intensity is more driven by the occurrence of the rainfall systems than by their own intensity. In Senegal, correlation between the return level map (d) and the proportion of rainfall days (b) is even larger. However the NE-SW gradient of return level maps (d) differs from the N-S gradient characterizing the mean number of rainfall days (b), while the mean of non-zero daily rainfall (c) displays a slight east-west distortion near the coast. This suggests that the spatial organization of extreme rainfall in Senegal might be a combined effect of rainfall occurrence and influence of oceanic moisture entries. This additional local oceanic moisture might also explain why extremes are more intense in Senegal than in the Central Sahel. 


\subsubsection{Co-occurrence of extremes}

Fig. 3 shows the empirical estimates of $\chi_{i j}(0.99)$, the conditional probability of coexceeding the $99 \%$-quantile, for all the pairs of stations $(i, j)$ lying in either region. The value of $p=0.99$ is chosen for illustration with the aim of being far enough in the tail of the distribution while having enough points to make robust empirical estimations. Remind that the $99 \%$ quantile corresponds almost to the 1-year return level in JJAS. Estimates are plotted as a function of the anisotropic distance (12) obtained from the estimated Brown-Resnick model of Section 3.2.2 (i.e. with the estimated matrix $M$ in (12)) for the 1950-2014 period. Each plot contains $N(N-1) / 2$ points corresponding to the number of pairs of stations. The scatter plot of empirical estimates versus anisotropic distance follows relatively well the curve of $\chi_{i j}(0.99)$ predicted by the estimated Brown-Resnick model, which is given by combining (9), (10), (12) and (13). Let us recall that the model is fitted on the rainfall data directly, and not on the empirical values of $\chi_{i j}(0.99)$ which are uncertain and are only shown here to help judging the quality of the fit. All in all, goodness-of-fit seems satisfactory. Furthermore, in the Central Sahel case, the empirical estimates of the AMMA-CATCH network are also relatively well aligned along the predicted curve, although the estimation is made independently of these data. However the estimated model tends to slightly underestimate the probability of conditional exceedances at very small distance. This may be due to the combination of several factors: first, the fact that AMMA-CATCH network covers a much smaller domain and dependence in the Niamey region may be slightly different than in the whole Central Sahel box. Related to this is the fact that few pairs are located at short distances in the Central Sahel network, and this may induce some bias for estimating the small scale dependence. Add to this the fact that AMMA-CATCH network covers only the most recent period (1990-2015) and cooccurrence of extremes may have changed since 1950. This question will be investigated in Section 4.2.

In order to allow comparison of the estimated probability of concomitant exceedances in the two region, we plot in Fig. 4 the values of $\chi_{i j}^{+}$as a function of the difference in coordinates $\left(s_{j}-s_{i}\right)$, in the two regions, adding the plot obtained for Niamey region from AMMA-CATCH network. Remind that $\chi_{i j}^{+}$is an approximate of the conditional probability of exceeding the $K$-year return level, for any $K \geq 1$ but $K \leq 100$ to embrace roughly the observed range of levels. For shortness, it is referred below as the conditional probability of concomitant large exceedances. As mentioned in Section 3.2.3, contours of 

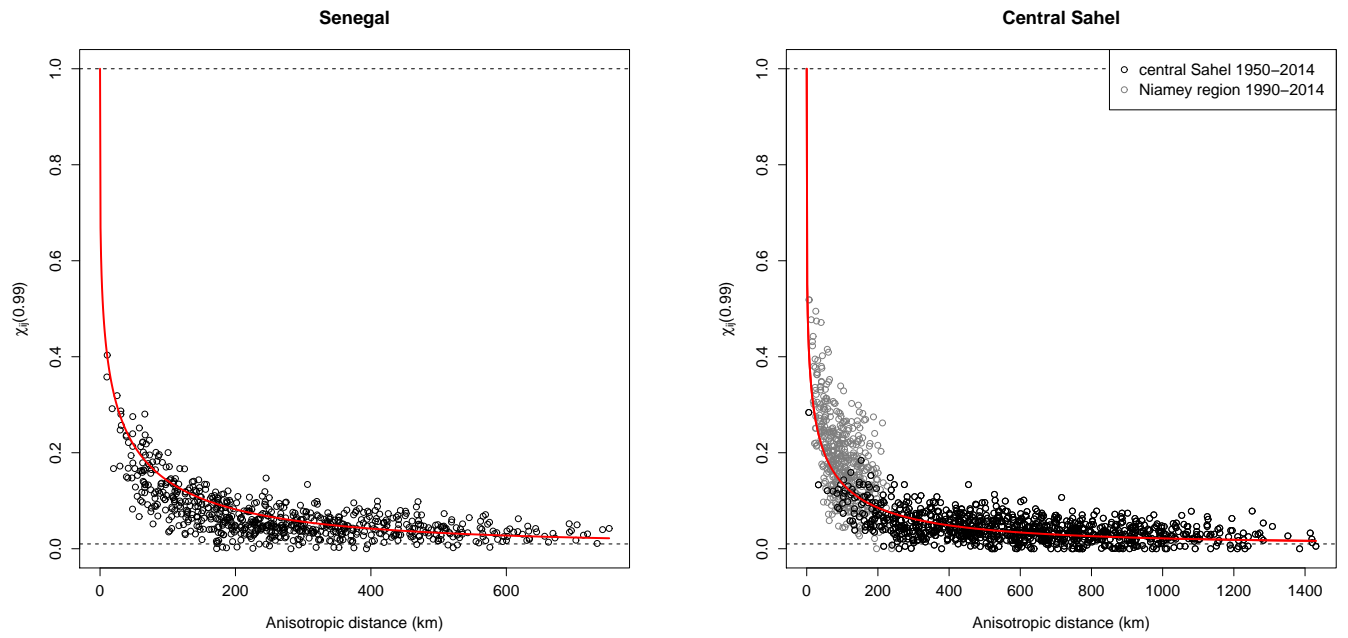

Figure 3. Empirical estimates of $\chi_{i j}(0.99)$, the conditional probability of co-exceeding the $99 \%$-quantile, for all pairs $(i, j)$ lying in (left) Senegal and (right) the Central Sahel, as a function of the anisotropic distance (12). The red curve shows the values predicted by the estimated Brown-Resnick process for the 1950-2014 period. The horizontal lines at abscissa 1 and 0.01 correspond respectively to cases of complete dependence and independence. In the Central Sahel case, the gray points show the empirical estimates obtained for the AMMA-CATCH network covering the period 1990-2014.

$\chi_{i j}^{+}=p$ delineate circles or ellipses, depending on whether dependence is respectively isotropic or anisotropic. Fig. 4 shows that a slight anisotropy is found in Senegal. The conditional probability of concomitant large exceedances is slightly larger in the E-50$\mathrm{N}$ direction (i.e. bearing $50^{\circ}$ anti-clockwise from East, with $\psi$ of (13) estimated to $50^{\circ}$ ). Anisotropy is more marked in the Central Sahel region with enhanced concomitance in the E-W direction $(\hat{\psi} \approx 0)$. At a given distance, Senegal shows a slightly larger probability of concomitance than the Central Sahel. For example there is on average $12 \%$ chance that a location in Senegal receives some extreme rainfall amount a day when extreme amount is received $100 \mathrm{~km}$ away (i.e. the average $\chi_{i j}^{+}$is 0.12 at $100 \mathrm{~km}$ distance). In the Central Sahel, there is on average $9 \%$ chance. In Niamey region, we find a marked anisotropy, as in the Central Sahel case, however the direction of maximum probability of concomitance is shifted to E-15-N. The dependence is also larger than in the entire Central Sahel region with e.g. on average $15 \%$ chance of conditional large exceedances at $100 \mathrm{~km}$ distance. As already mentioned, these differences may be due to the different domains, periods of observations and spatial scales the two networks allow to document. 

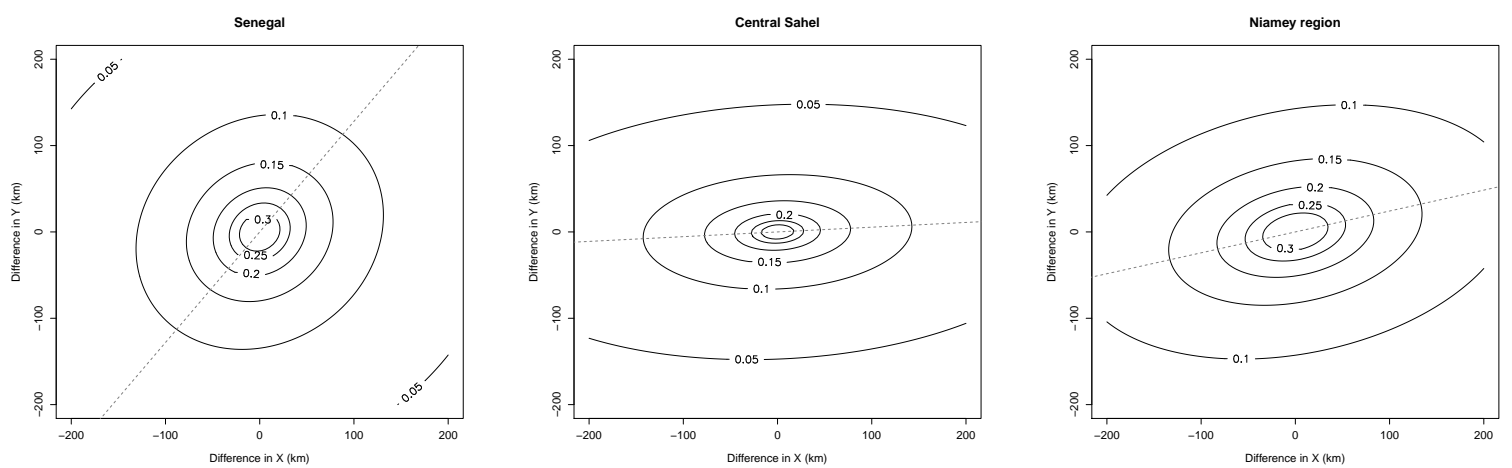

Figure 4. Values of $\chi_{i j}^{+}$, the conditional probability of concomitant large exceedances, under BrownResnick model, as a function of the difference in UTM coordinates $\left(s_{i}-s_{j}\right)$, for sites lying in (left) Senegal, (middle) the Central Sahel, and (right) the region of Niamey. In the latter case, estimation is based on the 1990-2014 period while in the two former cases it is based on the 1950-2014 period. The gray dotted lines show the direction of maximum conditional probability of large exceedances, given by $\psi$ of (13).

\subsection{Moving window case}

\subsubsection{Marginal distributions}

Fig. 5 shows the evolution of the regional averages of (a): mean annual rainfall, (b): mean proportion of rainfall days, (c): mean of non-zero daily rainfall and (d): 50-year return levels, estimated on 20-year moving windows from 1950-1969 to 1995-2014. The $95 \%$ confidence intervals are obtained from 200 bootstrap samples, resampling the days with replacement for each window and each station. Surprisingly contrasting results are found in Senegal and the Central Sahel for the mean values (a to c). In Senegal, three periods are found for the mean annual rainfall (a), with a decrease from the 50 s to the 70 s, followed by a relative stationarity till the $80 \mathrm{~s}$ and an increase since then. This is in line with the results of Lebel and Ali [2009] and Nicholson [2013]. The increase since the 80 s is due to an increase in both the proportion of wet days (b) and the mean rainfall during wet days (c). Despite the increase in the last decades, the levels of the mean values (a to c) at the end of the period have barely reached the stationary levels. In the Central Sahel, two periods are mainly found for the mean values (a to c). A decrease is found from the 50s to the 70s, as in Senegal, but followed by an increase since the 70s, which is particularly strong for the mean rainfall during wet days (c). 
(a)
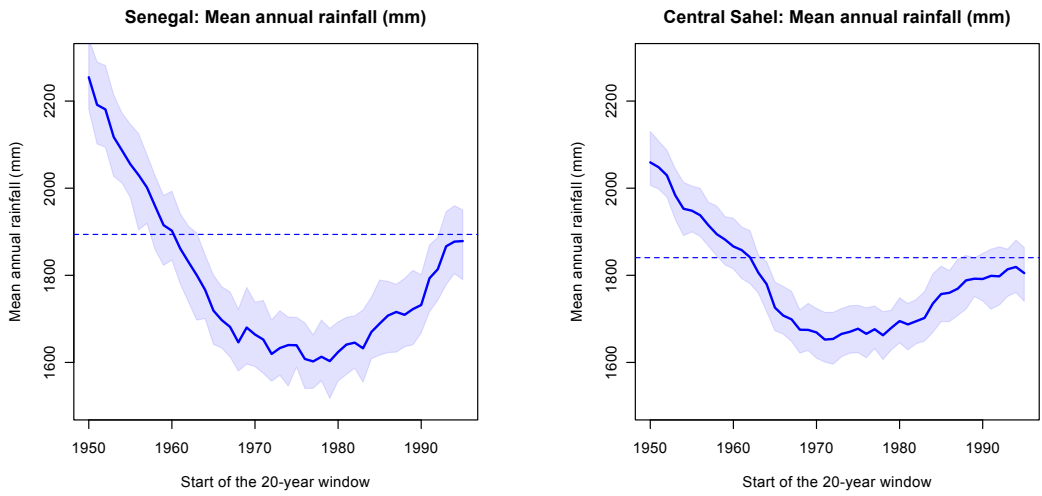

(b)
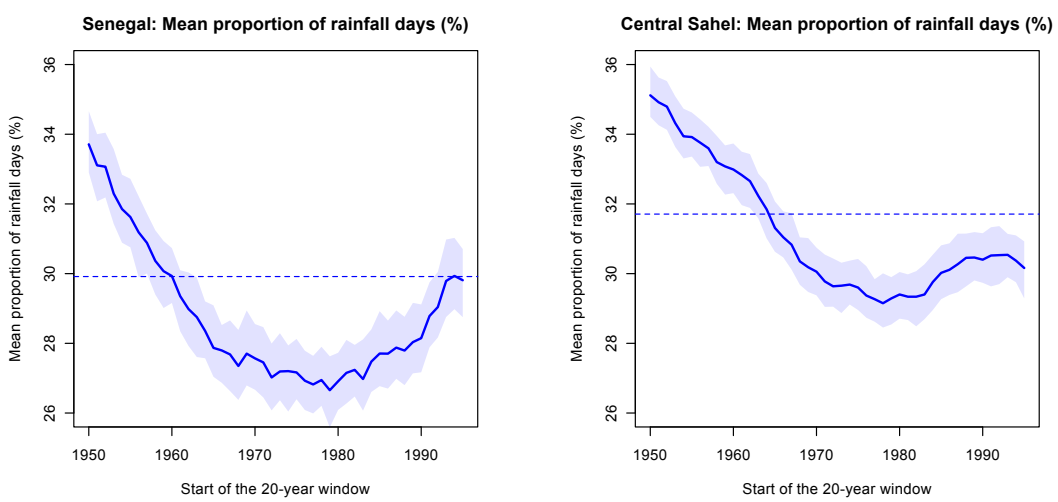

(c)
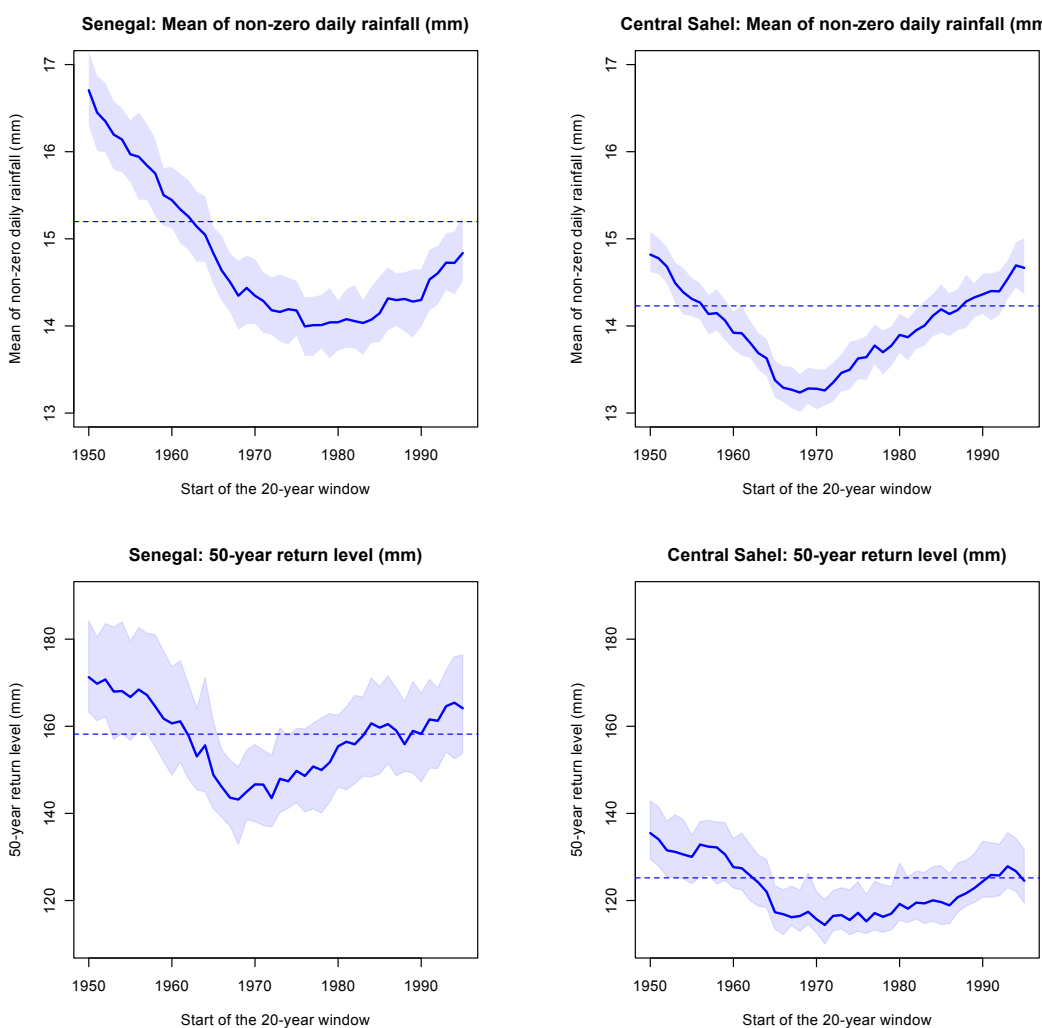

Figure 5. Regional averages of (a): mean annual rainfall, (b): mean proportion of rainfall days, (c): mean of non-zero daily rainfall and (d): 50-year return levels, estimated on 20-year moving windows from 19501969 to 1995-2014, in (left) Senegal, and (right) the Central Sahel. The dashed horizontal lines show the averages obtained over the whole period. The colored pands show the 95\%-confidence intervals obtained from 200 bootstrap samples. 
For the 50-year return level (d), similar evolutions are found for the two regions, with two main regimes. First, a decrease between the 50s and the 70s, with return levels $15 \%$ smaller in $1968-1977$ than they used to be in $1950-1969$. This period of decrease is followed by a period of increase in both regions since the 70 s- 80 s, particularly in Senegal. The 50-year return levels in Senegal have exceeded the stationary levels since the 80 s and almost reached the levels of the beginning of the period. In the Central Sahel, the stationary levels have barely been recovered so far. These results on extreme rainfalls evolution in the Central Sahel are coherent with Panthou et al. [2013] who found a significant decrease of around 10\% in the 2-year return level of the periods 1950-1969 and 1970-1990. Over the recent decade, the evolution of the $K$-year return level in the Central Sahel is also in line with the upward trend in daily rainfall maxima shown by Panthou et al. [2014].

\subsubsection{Co-occurrence of extremes}

Fig. 6 shows the ellipses of the contours $\left\{\chi_{i j}^{+}=0.1\right\}$ predicted by the BrownResnick model estimated on 20-year moving windows for the two regions. Thus each ellipse delineates the set of points having $10 \%$ chance of exceeding some extreme level a day when some extreme level is exceeded in the center of the ellipses. To better assess changes in these ellipses, we also plot in Fig. 6 the length of the minor and major axis of the ellipses. In order to assess uncertainty in these values, a bootstrap method is applied. For each window and each region, i) we sample the days with replacement; ii) we estimate the marginal GPD distributions on the bootstrap data exceeding the threshold $u_{j}$ used for the original data (thus the threshold itself does not change along the bootstrap procedure); iii) we transform the data exceeding the threshold into unit-Fréchet variates; iv) we fit the anisotropic Brown-Resnick model on these new data. We apply this 200 times, giving 200 bootstrap estimations for each window. The confidence bands in Fig. 6 show the 0.025and 0.975 -quantile of these estimations.

In Senegal, concomitance for the periods $1950-1969$ to $1975-1994$ is characterized by a preferred direction in the E-50-N direction, which is also the direction found in the stationary case (see Section 4.1.2). However concomitance of the largest rainfalls along this direction was more likely during this period than over all 1950-2014, as shown by the major axis compared to the stationary case in Fig. 6. This anisotropy can be considered as 
relatively significant since there is little overlap of the $95 \%$ confidence bands of the major and minor axis lengths.

For the periods 1976-1995 to 1981-2000, ellipses are less and less elongated, which means that within this period, the preferred direction of concomitance vanished. Finally, from 1982-2001 to 1995-2014, concomitance is characterized by a stability in the shape of the ellipses which are roughly isotropic (i.e. the lengths of the major and minor axes are equal). Thus since the 80 s concomitance of extreme rainfall is as likely in any direction but weaker than it used to be, as shown by the red ellipses of Fig. 6 .

In the Central Sahel, the periods from 1950-1969 to 1981-2000 are characterized by a relative stability in the shape of the ellipses of co-occurrence, with a preferred direction in roughly the E-W direction, as in the stationary case of Section 4.1.2. Judging by the confidence bands in Fig. 6, this preferred direction is highly significant. As for the Senegal, a change in the shape of the ellipses is found in the 80s but surprisingly with opposite consequences. The period from 1982-2001 to 1990-2014 is characterized by a strong enlargement of the ellipses of Fig. 6 in all directions, meaning that, contrary to Senegal, co-occurrence tends to be significantly stronger in all directions these last years.

\section{Conclusions and Discussion}

In this paper we study the co-occurrence of extreme daily rainfall and its evolution since 1950 in two Sahelian regions: the Western (Senegal) and the Central Sahel. Our approach is based on a max-stable modeling of rainfall threshold exceedances. This allows us to quantify the probability of experiencing concomitant extremes in these regions and to document, in a moving window approach, whether and to what extend this probability has evolved since 1950.

Some features of the evolution of extreme rainfall regime appear to be consistent for both studied Sahelian regions. They concern the evolution of the marginal distributions of extreme daily rainfall. A change in the 80 s is found with a period of decreasing rainfall intensity between the $50 \mathrm{~s}$ and the $70 \mathrm{~s}$, followed by a period of increasing intensity since the 80s. This confirms previous results found for the Central Sahel by Panthou et al. [2013] and Panthou et al. [2014]. It is also in line with the increasing trend in extreme rainfall detected since 1982 by Taylor et al. [2017] over the entire Sahel. However some results show contrasted evolutions between Senegal and the Central Sahel. In Sene- 

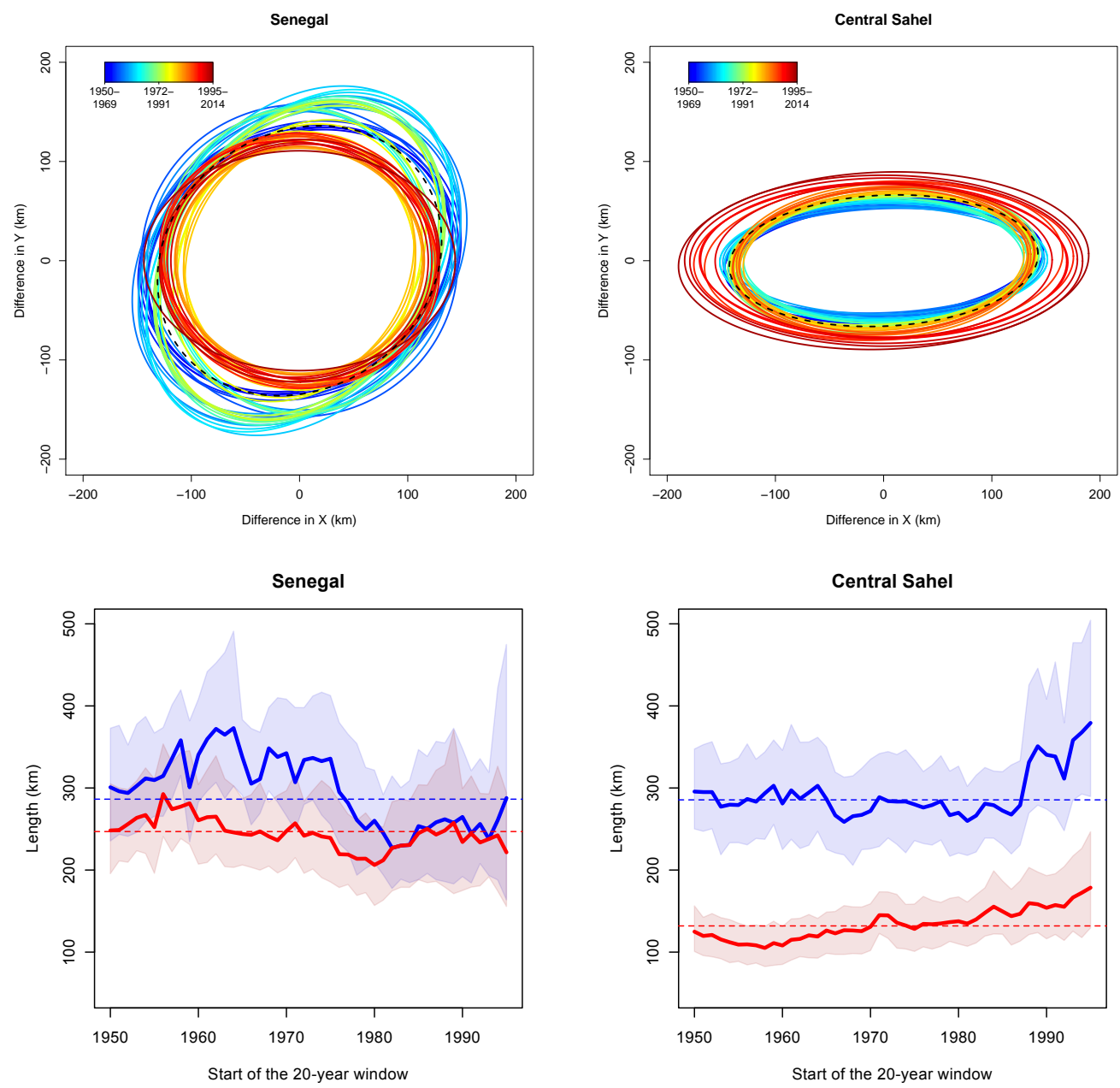

Figure 6. Top: Contours of $\left\{\chi_{i j}^{+}=0.1\right\}$ estimated on 20-year moving windows in (left) Senegal, and (right) the Central Sahel. The dotted black ellipses are the contours obtained in the stationary case (see Fig. 4). Bottom: Length of the major (blue) and minor (red) axes of the ellipses delineating the contours $\left\{\chi_{i j}^{+}=0.1\right\}$, as a function of the starting year of the 20-year moving windows. The bands show the $95 \%$ confidence intervals obtained for 200 bootstrap samples. The dotted horizontal lines are the values obtained in the stationary case. 
gal, a change is found in the $80 \mathrm{~s}$ in the size and direction of the ellipses delineating the contours of co-occurrence at extreme levels, with preferred co-occurrence in the direction E-50-N before the 80s, and isotropic co-occurrence with lesser extent afterwards. In the Central Sahel, a change is also found in the 80s but with opposite consequences. Ellipses of co-occurrence show a greater extent after the 80s, while anisotropy remains in the E-W direction over the whole period.

These contrasts raise several questions about the evolution of extreme precipitation systems. In the Sahel, most of the rainfall is produced by Mesoscale Convective Systems (MCSs). MCSs are often organized in squall lines propagating from East to West. Under particular conditions, organized convective systems can be stationary (long-lasting systems) and may produce large rainfall amounts over a particular location [Lafore et al., 2017; Vischel et al., 2017]. Local convection can also occur, but this generally produces less rainfall. We refer to Laurent et al. [1998] and Mathon et al. [2002] for a review of MCSs climatology in the Sahel.

The observed evolution of the ellipses of co-occurrence in extremes can be interpreted in light of a change in typology of extreme precipitation systems, assuming some link between the two of them. First, one can postulate that anisotropic ellipses correspond to propagative systems, while isotropic ellipses correspond to stationary systems, and that the more elongated the ellipses, the more propagative the system. Second, larger probabilities of concomitant extremes (larger minor axis and/or bigger ellipses) can be expected to come from bigger extreme rainfall systems.

If these assumptions hold, then the increase in the size of the ellipses since the 80s in the Central Sahel would be a consequence of more propagative and slightly bigger MCSs. This is a plausible hypothesis since the more organized systems in this region [Mesoscale Convective Complex, as defined by Mathon et al., 2002] have such spatial characteristics and are very rainy efficient [Laurent et al., 1998; Mathon et al., 2002]. The way we interpret the changes in ellipse features is supported by the recent results of Taylor et al. [2017]. They show from Infra-Red (IR) satellite data (period 1982-2016) that the trend in extreme rainfall is associated with a significant increasing trend in the vertical development of the largest Sahelian MCSs, that are known to propagate faster, and to a smaller extent with a trend in their horizontal extension. This evolution of MCS features 
is retrieved in Fig. 6 where the trend in the major axis of ellipses in the Central Sahel is more pronounced than the trend in their minor axis.

Following the same reasoning in Senegal, the transition between anisotropic to isotropic ellipses could be the manifestation of (i) a transition between a large proportion of propagative systems to a large proportion of stationary systems, (ii) a more erratic propagative direction of systems, and/or (iii) more localized but intense convection.

The reasons for such a contrasted evolution of extreme rainy system features between Senegal and the Central Sahel still have to be determined. The main drivers of the rainfall intensification in the Sahel have been recently identified by Taylor et al. [2017]. They incriminate the warming of the Saharan (in link to anthropogenic GHG emissions) since it intensifies convection within MCSs through increased wind shear and intrusion of the hot and dry Saharan air layer at the mid-troposphere. These factors tend to decrease the occurrence of small and localized systems (due to higher convective energy barriers initiating convection) but favor higher development and stronger storms once convection is initiated. While these processes support our results in the Central Sahel, they do not directly explain why storm properties evolved differently in the Western Sahel. Lebel and Ali [2009] have pointed out the presence of an East-West contrast over the period 19902007 with a Western Sahel remaining dryer than the Central Sahel for which a relative increase of mean annual rainfall was observed. This zonal rainfall dipole is also a consistent pattern in GCM rainfall projections [Monerie et al., 2012; Gaetani et al., 2017] and is expected to accentuate over the 21 st century [James et al., 2015]. The warming of the Sahara is also thought to be responsible for this contrast especially through the enhancement of the Saharan Heat Low activity which simultaneously favors air subsidence in the West - preventing deep moist convection - and accentuates monsoon fluxes in the Central Sahel [Monerie et al., 2012; James et al., 2015; Lavaysse et al., 2015].

However the way these changes in monsoon circulation might affect extreme rainy systems properties differently in the West and the Central Sahel remains an open question. In addition to these synoptic features, more local processes due to land-ocean interface can also not be excluded to have an influence on storm development and propagation.

While the main objective of the present paper was to apply a modern statistical frameworks to characterize the extreme rainfall co-occurrence, the way we interpret the results in light of changes in storm features and atmospheric environment calls for further 
research. Next work will be to use other datasets from which MCS properties can be inferred to confirm the contrasted trends in MCS properties in Senegal and Central Sahel. To that end, the direct analysis of brightness temperature from IR satellite data as in Taylor et al. [2017] or the analysis of IR-derived MCS tracking products [as those proposed by Fiolleau and Roca, 2013] might be considered. Further work is also required to better understand the atmospheric mechanisms responsible for the contrasted changes in storms properties. A first option to that end is to make an analytic approach similar to that proposed by Taylor et al. [2017], including the analysis of regional contrasts in storms properties within the Sahel. A second possibility is to extend the case studies of extreme events such as Lafore et al. [2017] and Vischel et al. [2017] in Ouagadougou to other locations in the Sahel [see e.g. Engel et al., 2017]. Atmospheric simulation experiments, in particular high-resolution convection-permitting simulations, could also be very helpful to find some physical explanations of the observed trends in the co-occurrence of extremes.

\section{Acknowledgments}

The research leading to these results has received funding from the NERC/DFID Future Climate For Africa programme under the AMMA-2050 project, grant numbers NE/M020428/1. This work was also supported by the French national programme EC2CO-LEFE "Recent evolution of hydro-climatic hazards in the Sahel: detection and attribution". The AMMACATCH rainfall data are freely available at http://bd.amma-catch.org/ except for the last three years which are subject to restricted access. The other daily data are subject to access restrictions according to the data policy defined by the National Weather Services in Senegal, Burkina Faso, Niger, Benin and Togo and are not freely available.

\section{References}

Ali, A., T. Lebel, and A. Amani (2003), Invariance in the Spatial Structure of Sahelian Rain Fields at Climatological Scales, Journal of Hydrometeorology, 4, 996-1011.

Ali, A., A. Amani, T. Lebel, and S. Ibrahima (2006), Utilisation optimale de l'information pluviométrique des MCGA aux échelles hydrologiques au Sahel = Optimal use of GCM rainfall information at the hydrological scale in the Sahel, in Climate Variability and Change - Hydrological Impacts, pp. 430-435, International Association of Hydrological Sciences press, Wallingford, ROYAUME-UNI, Havana, Cuba. 
Asadi, P., A. C. Davison, and S. Engelke (2015), Extremes on river networks, Ann. Appl. Stat., 9(4), 2023-2050, doi:10.1214/15-AOAS863.

Bacro, J.-N., and C. Gaetan (2014), Estimation of spatial max-stable models using threshold exceedances, Statistics and Computing, 24(4), 651-662, doi:10.1007/s11222-0139394-4.

Bell, M. A., and P. J. Lamb (2006), Integration of weather system variability to multidecadal regional climate change: The West African Sudan-Sahel zone, 1951-98, Journal of Climate, 19(20), 5343-5365.

Blanchet, J., and A. C. Davison (2011), Spatial modeling of extreme snow depth, Annals of Applied Statistics, 5(3), 1699-1725.

Castruccio, S., R. Huser, and M. G. Genton (2016), High-Order Composite Likelihood Inference for Max-Stable Distributions and Processes, Journal of Computational and Graphical Statistics, 25(4), 1212-1229, doi:10.1080/10618600.2015.1086656.

Ceresetti, D., S. Anquetin, G. Molinié, E. Leblois, and J.-D. Creutin (2012), Multiscale Evaluation of Extreme Rainfall Event Predictions Using Severity Diagrams, Weather and Forecasting, 27, 174-188, doi:10.1175/WAF-D-11-00003.1.

Coles, S. (2001), An introduction to statistical modeling of extreme values, Springer Series in Statistics, Springer-Verlag, London.

Coles, S., J. Heffernan, and J. Tawn (1999), Dependence Measures for Extreme Value Analyses, Extremes, 2(4), 339-365, doi:10.1023/A:1009963131610.

Dai, A., P. J. Lamb, K. E. Trenberth, M. Hulme, P. D. Jones, and P. Xie (2004), The recent Sahel drought is real, International Journal of Climatology, 24(11), 1323-1331, doi:10.1002/joc.1083.

Davison, A. C., and M. M. Gholamrezaee (2011), Geostatistics of extremes, Proceedings of the Royal Society of London A: Mathematical, Physical and Engineering Sciences, doi: 10.1098/rspa.2011.0412.

Davison, A. C., S. A. Padoan, and M. Ribatet (2012), Statistical Modeling of Spatial Extremes, Statistical Science, 27(2), 161-186, doi:10.1214/11-STS376.

Davison, A. C., R. Huser, and E. Thibaud (2013), Geostatistics of Dependent and Asymptotically Independent Extremes, Mathematical Geosciences, 45(5), 511-529, doi: 10.1007/s11004-013-9469-y.

de Haan, L., and A. Ferreira (2006), Extreme Value Theory: An Introduction, Springer Series in Operations Research and Financial Engineering, 1 ed., Springer-Verlag New 
York, doi:10.1007/0-387-34471-3.

De Michele, C., N. Kottegoda, and R. Rosso (2002), IDAF (Intensity-duration-areafrequency) curves of extreme storm rainfall: a scaling approach, Water Science and Technology, 45(2), 83-90.

Delrieu, G., A. Wijbrans, B. Boudevillain, D. Faure, L. Bonnifait, and P.-E. Kirstetter (2014), Geostatistical radarâĂŞraingauge merging: A novel method for the quantification of rain estimation accuracy, Advances in Water Resources, 71, 110 - 124, doi: http://dx.doi.org/10.1016/j.advwatres.2014.06.005.

Di Baldassarre, G., A. Montanari, H. Lins, D. Koutsoyiannis, L. Brandimarte, and G. BlÂúschl (2010), Flood fatalities in Africa: From diagnosis to mitigation, Geophysical Research Letters, 37(22), doi:10.1029/2010GL045467.

Dombry, C., and M. Ribatet (2015), Functional regular variations, Pareto processes and peaks over threshold, Statistics and Its Interface, 8(1), doi: http://dx.doi.org/10.4310/SII.2015.v8.n1.a2.

Engel, T., A. H. Fink, P. Knippertz, G. Pante, and J. Bliefernicht (2017), Extreme precipitation in the west african cities of dakar and ouagadougou - atmospheric dynamics and implications for flood risk assessments, Journal of Hydrometeorology, doi:10.1175/JHMD-16-0218.1.

Ferreira, A., and L. de Haan (2014), The generalized Pareto process; with a view towards application and simulation, Bernoulli, 20(4), 1717-1737, doi:10.3150/13-BEJ538.

Ferro, C. A. T., and J. Segers (2003), Inference for Clusters of Extreme Values, Journal of the Royal Statistical Society. Series B (Statistical Methodology), 65(2), pp. 545-556.

Fiolleau, T., and R. Roca (2013), An Algorithm for the Detection and Tracking of Tropical Mesoscale Convective Systems Using Infrared Images From Geostationary Satellite, IEEE Transactions on Geoscience and Remote Sensing, 51(7), 4302-4315, doi: 10.1109/TGRS.2012.2227762.

Gaetani, M., C. Flamant, S. Bastin, S. Janicot, C. Lavaysse, F. Hourdin, P. Braconnot, and S. Bony (2017), West African monsoon dynamics and precipitation: the competition between global SST warming and CO2 increase in CMIP5 idealized simulations, Climate Dynamics, 48(3), 1353-1373, doi:10.1007/s00382-016-3146-z.

Gallego, D., P. Ordóñez, P. Ribera, C. Peña-Ortiz, and R. García-Herrera (2015), An instrumental index of the West African Monsoon back to the nineteenth century: An Instrumental Index of the West African Monsoon, Quarterly Journal of the Royal Meteoro- 
logical Society, 141(693), 3166-3176, doi:10.1002/qj.2601.

Gaume, J., N. Eckert, G. Chambon, M. Naaim, and L. Bel (2013), Mapping extreme snowfalls in the French Alps using max-stable processes, Water Resources Research, 49(2), 1079-1098, doi:10.1002/wrcr.20083.

Gerbaux, M., N. Hall, N. Dessay, and I. Zin (2009), The sensitivity of Sahelian runoff to climate change / Sensibilite au changement climatique du ruissellement au Sahel, Hydrological Sciences Journal, 54(1), 5-16, doi:10.1623/hysj.54.1.5.

Hulme, M., R. Doherty, T. Ngara, M. New, and D. Lister (2001), African climate change: 1900-2100, Climate Research, 17(2), 145-168.

Huser, R., and A. C. Davison (2013), Composite likelihood estimation for the BrownResnick process, Biometrika, 100(2), 511-518, doi:10.1093/biomet/ass089.

Huser, R., and A. C. Davison (2014), Space-time modelling of extreme events, Journal of the Royal Statistical Society: Series B (Statistical Methodology), 76(2), 439-461, doi: 10.1111/rssb.12035.

James, R., R. Washington, and R. Jones (2015), Process-based assessment of an ensemble of climate projections for West Africa, Journal of Geophysical Research: Atmospheres, 120(4), 1221-1238, doi:10.1002/2014JD022513.

Kabluchko, Z., M. Schlather, and L. d. Haan (2009), Stationary Max-Stable Fields Associated to Negative Definite Functions, The Annals of Probability, 37(5), pp. 2042-2065.

Lafore, J.-P., F. Beucher, P. Peyrillé, A. Diongue-Niang, N. Chapelon, D. Bouniol, G. Caniaux, F. Favot, F. Ferry, F. Guichard, E. Poan, R. Roehrig, and T. Vischel (2017), A multi-scale analysis of the extreme rain event of ouagadougou in 2009, Quarterly Journal of the Royal Meteorological Society, doi:10.1002/qj.3165, qJ-17-0033.R2.

Laurent, H., N. D'Amato, and T. Lebel (1998), How Important is the Contribution of the Mesoscale Convective Complexes to the Sahelian Rainfall?, Journal of Physics and Chemistry of The Earth, 23, 629-633.

Lavaysse, C., C. Flamant, A. Evan, S. Janicot, and M. Gaetani (2015), Recent climatological trend of the Saharan heat low and its impact on the West African climate, Climate Dynamics, pp. 1-20, doi:10.1007/s00382-015-2847-z.

Le Barbé, L., and T. Lebel (1997), Rainfall climatology of the HAPEX-Sahel region during the years 1950-1990, Journal of Hydrology, 188-189, 43-73.

Le Barbé, L., T. Lebel, and D. Tapsoba (2002), Rainfall Variability in West Africa during the Years 1950-90, Journal of Climate, 15, 187-202. 
Lebel, T., and A. Ali (2009), Recent trends in the Central and Western Sahel rainfall regime (1990-2007), Journal of Hydrology, 375(1-2), 52-64.

Lebel, T., A. Diedhiou, and H. Laurent (2003), Seasonal cycle and interannual variability of the Sahelian rainfall at hydrological scales, Journal Of Geophysical Research, 108, doi:10.1029/2001JD001,580.

Lebel, T., B. Cappelaere, S. Galle, N. Hanan, L. Kergoat, S. Levis, B. Vieux, L. Descroix, M. Gosset, E. Mougin, C. Peugeot, and L. Seguis (2009), AMMA-CATCH studies in the Sahelian region of West-Africa: An overview, Journal of Hydrology, 375(1âĂŞ2), 3 - 13, doi:https://doi.org/10.1016/j.jhydrol.2009.03.020, surface processes and water cycle in West Africa, studied from the AMMA-CATCH observing system.

Mathon, V., H. Laurent, and T. Lebel (2002), Mesoscale Convective System Rainfall in the Sahel, Journal of Applied Meteorology, 41, 1081-1092.

Monerie, P.-A., B. Fontaine, and P. Roucou (2012), Expected future changes in the African monsoon between 2030 and 2070 using some CMIP3 and CMIP5 models under a medium-low RCP scenario, Journal of Geophysical Research: Atmospheres, 117(D16), n/a-n/a, doi:10.1029/2012JD017510.

Nicholson, S. (2005), On the question of the âĂIJrecoveryâĂİ of the rains in the West African Sahel, Journal of Arid Environments, 63(3), 615 - 641, doi: https://doi.org/10.1016/j.jaridenv.2005.03.004, special Issue on The Greening of the Sahel.

Nicholson, S. E. (2013), The West African Sahel: A Review of Recent Studies on the Rainfall Regime and Its Interannual Variability, ISRN Meteorology, 2013, 1-32, doi: $10.1155 / 2013 / 453521$.

Nicolet, G., N. Eckert, S. Morin, and J. Blanchet (2016), Decreasing spatial dependence in extreme snowfall in the French Alps since 1958 under climate change, Journal of Geophysical Research: Atmospheres, pp. n/a-n/a, doi:10.1002/2016JD025427, 2016JD025427.

Nicolet, G., N. Eckert, S. Morin, and J. Blanchet (2017), A multi-criteria leave-two-out cross-validation procedure for max-stable process selection, Spatial Statistics, 22(Part 1), 107 - 128, doi:https://doi.org/10.1016/j.spasta.2017.09.004.

Opitz, T. (2013), Extremal processes: Elliptical domain of attraction and a spectral representation, Journal of Multivariate Analysis, 122, 409 - 413, doi: http://dx.doi.org/10.1016/j.jmva.2013.08.008. 
Padoan, S. A., M. Ribatet, and S. A. Sisson (2010), Likelihood-Based Inference for MaxStable Processes, Journal of the American Statistical Association, 105(489), 263-277, doi:10.1198/jasa.2009.tm08577.

Panthou, G., T. Vischel, T. Lebel, J. Blanchet, G. Quantin, and A. Ali (2012), Extreme rainfall in West Africa: A regional modeling, Water Resources Research, 48, W08,501, doi:10.1029/2012WR012052.

Panthou, G., T. Vischel, T. Lebel, G. Quantin, A.-C. F. Pugin, J. Blanchet, and A. Ali (2013), From pointwise testing to a regional vision: An integrated statistical approach to detect nonstationarity in extreme daily rainfall. Application to the Sahelian region, Journal of Geophysical Research: Atmospheres, 118(15), 8222-8237, doi: 10.1002/jgrd.50340.

Panthou, G., T. Vischel, T. Lebel, G. Quantin, and G. Molinié (2014), Characterising the space-time structure of rainfall in the Sahel with a view to estimating IDAF curves, $H y$ drology and Earth System Sciences, 18(12), 5093-5107, doi:10.5194/hess-18-5093-2014.

Raillard, N., P. Ailliot, and J. Yao (2014), Modeling extreme values of processes observed at irregular time steps: Application to significant wave height, Ann. Appl. Stat., 8(1), 622-647, doi:10.1214/13-AOAS711.

Reich, B. J., B. A. Shaby, and D. Cooley (2014), A Hierarchical Model for SeriallyDependent Extremes: A Study of Heat Waves in the Western US, Journal of Agricultural, Biological, and Environmental Statistics, 19(1), 119-135, doi:10.1007/s13253-0130161-y.

Sanogo, S., A. H. Fink, J. A. Omotosho, A. Ba, R. Redl, and V. Ermert (2015), Spatiotemporal characteristics of the recent rainfall recovery in West Africa, International Journal of Climatology, pp. n/a-n/a, doi:10.1002/joc.4309.

Schlather, M. (2002), Models for Stationary Max-Stable Random Fields, Extremes, 5(1), 33-44, doi:10.1023/A:1020977924878.

Shaby, B. A., and B. J. Reich (2012), Bayesian spatial extreme value analysis to assess the changing risk of concurrent high temperatures across large portions of European cropland, Environmetrics, 23(8), 638-648, doi:10.1002/env.2178.

Shang, H., J. Yan, and X. Zhang (2011), El Niño-Southern Oscillation influence on winter maximum daily precipitation in California in a spatial model, Water Resources Research, 47(11), n/a-n/a, doi:10.1029/2011WR010415, w11507. 
Tarhule, A. (2005), Damaging Rainfall and Flooding: The Other Sahel Hazards, Climatic Change, 72(3), 355-377, doi:10.1007/s10584-005-6792-4.

Taylor, C., D. Belusic, F. Guichard, D. Parker, T. Vischel, O. Bock, P. Harris, S. Janicot, C. Klein, and G. Panthou (2017), Frequency of extreme Sahelian storms tripled since 1982 in satellite observations, Nature, 544(7651), 475-478, doi:10.1038/nature22069.

Thibaud, E., and T. Opitz (2015), Efficient inference and simulation for elliptical Pareto processes, Biometrika, 102(4), 855-870, doi:10.1093/biomet/asv045.

Thibaud, E., R. Mutzner, and A. C. Davison (2013), Threshold modeling of extreme spatial rainfall, Water Resources Research, 49(8), 4633-4644, doi:10.1002/wrcr.20329.

Varin, C., N. Reid, and D. Firth (2011), AN OVERVIEW OF COMPOSITE LIKELIHOOD METHODS, Statistica Sinica, 2l(1), 5-42.

Vischel, T., and T. Lebel (2007), Assessing the water balance in the Sahel: Impact of small scale rainfall variability on runoff. Part 2: Idealized modeling of runoff sensitivity, Journal of Hydrology, 333(2-4), 340-355.

Vischel, T., G. Panthou, P. Peyrillé, R. Roehrig, G. Quantin, T. Lebel, C. Wilcox, F. Beucher, and M. Budiarti (2017), Precipitation extremes in the West African Sahel: recent evolution and physical mechanisms, in Tropical Climate Extremes: Natural Variability and Trends, Synthèses, elsevier ed.

Wadsworth, J. L., and J. A. Tawn (2012), Dependence modelling for spatial extremes, Biometrika, 99(2), 253-272, doi:10.1093/biomet/asr080.

Wadsworth, J. L., and J. A. Tawn (2014), Efficient inference for spatial extreme value processes associated to log-Gaussian random functions, Biometrika, 101(1), 1-15, doi: 10.1093/biomet/ast042.

Westra, S., and S. A. Sisson (2011), Detection of non-stationarity in precipitation extremes using a max-stable process model, Journal of Hydrology, 406(1-2), 119-128, doi:http://dx.doi.org/10.1016/j.jhydrol.2011.06.014.

Zhang, Q., M. Xiao, V. P. Singh, and Y. D. Chen (2014), Max-stable based evaluation of impacts of climate indices on extreme precipitation processes across the Poyang Lake basin, China, Global and Planetary Change, 122, 271 - 281, doi: https://doi.org/10.1016/j.gloplacha.2014.09.005. 